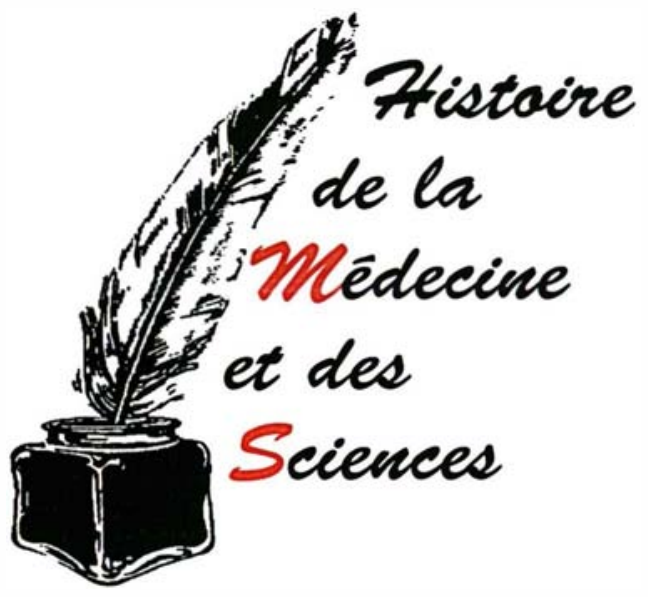

A Ifred Marie-François Donne, né à Noyon dans l'Oise le 13 Vendémiaire de l'an X (13 septembre 1801), fils de négociant, vint à Paris à l'âge de 20 ans et, suivant la demande de sa famille, fit des études de Droit. N'ayant, à l'expérience, aucun goût pour cette discipline, il débuta des études de médecine à 25 ans, présenta sa thèse à 30 ans et fut rapidement Chef de Clinique de Bouillaud* et de Louis*. Ayant l'avantage d'être fortuné, il pouvait se permettre de faire un peu ce qui lui plaisait. Il était probablement " le seul étudiant de son temps qui vint à l'école en voiture" (Daremberg). Esprit ingénieux et chercheur ouvert et curieux, très rapidement il s'employa à appliquer les Sciences à la Médecine. Il utilisa le thermomètre pour mesurer la température des malades, contrairement à Chomel, Chef de Service de l'Hôtel Dieu qui préférait l'évaluation tactile de la température, et confronta la température à la fréquence du pouls et de la respiration. Il se servit du papier de tournesol pour étudier l'acidité ou l'alcalinité de nombreux liquides de l'organisme et, surtout, exploita toute la richesse d'un mon-

* Deux chefs de service de Paris à qui l'on doil notamment la description du RAA (Bouillaud) et la percussion du thorax (angle de louis dans l'aire pré-

\title{
Un pionnier de la médecine scientifique, Alfred Donne
}

de nouveau vu par le microscope : sa Thèse de Médecine "Recherches physiologiques et chimicomicroscopiques sur les globules du sang, du pus, du mucus et sur ceux de l'humeur de l'œil ", soutenue en 1831, marque le début d'une nouvelle ère d'application des sciences exactes en médecine.

A cette époque, De Cabanis pensait qu'il fallait " se débarrasser des objets inutiles : la médecine est une science surchargée de bagages surabondants ", Trousseau rejetait l'idée de pathologie cellulaire : " elle fait oublier l'homme pour ne songer qu'aux cellules et elle s'y perd dans l'abîme des infiniments petits ". Bichat n'utilisait pas le microscope et Broussais prétendait que toutes les maladies dérivaient de l'irritation de l'estomac.

Seuls les liquides pouvaient être étalés et vus au microscope. Alfred Donne décrivit les images du lait, du sang et de différents liquides. Il avait le culte du lait et mit toute son énergie à reconnaître le bon lait du mauvais. Ses descriptions les plus précises furent cependant celles des globules du sang. Par une étude méthodique, il prouva la réalité des globules dans le sang et entra en conflit avec les maîtres de l'époque : en 1827 , Magendie écrivait dans son Précis élémentaire de Physiologie: "Je crois qu'il s'est glissé beaucoup d'imagination, d'erreurs, d'illusions optiques dans toutes ces opinions diverses. J'ai fait un grand nombre d'expériences microscopiques pour m'éclairer à cet égard : je n'ai jamais vu dans le sang de l'homme que des particules de matières colorantes, ordinairement arrondies, de différentes grosseurs qui, suivant qu'elles sont placées exactement ou inexactement au foyer du microscope, paraissent tantôt plates, tantôt sphériques et d'autres fois ayant la figure d'un disque percé au centre : toutes ces apparences se produisent à volonté en variant la position des particules relativement à l'instrument. Je crois aussi qu'on a souvent décrit et dessiné dans les ouvrages des bulles d'air pour des globules de sang. " Raspail lui aussi contestait la présence de globules dans le sang mais, en revanche, voyait des éléments figurés dans le lait, ce que réfuta, là encore, Alfred Donne qui prouva qu'ils étaient " simplement formés de matières grasses agglomérées"(Daremberg). Si les travaux d'Alfred Donne étaient appréciés par l'Académie des Sciences - il fut d'ailleurs l'un des premiers journalistes scientifiques, assurant régulièrement, à partir de 1829, une chronique du Journal des Débats dans laquelle il rendait compte des séances de l'Académie des Sciences - ils étaient, en revanche, reçus avec un grand scepticisme de la 
part des Maîtres de la Faculté de Médecine. Alfred Donne échoua à deux concours d'agrégation de médecine, en 1832 et en 1835 . Nombreux étaient en effet les adversaires de l'emploi du microscope auquel on attribuait des illusions d'optique; de plus, comme le disait Léon Rostand en 1831, beaucoup pensaient que " c'est au lit du malade que tombent tous les vains systèmes, toutes les théories, toutes les hypothèses ".

Alfred Donne organisa, à ses frais, un cours de microscopie clinique qui connut une grande affluence et fit concurrence à la Faculté. Il donna des cours et organisa des travaux pratiques jusqu'en 1845. Il transforma le microscope à lumière solaire en un microscope utilisant la lumière artificielle grâce à un mélange de gaz (lumière oxhydrique). Léon Foucault, un de ses assistants, devint physicien et collabora à la confection de photographies daguerréotypes de préparations microscopiques, présentées pour la première fois le 24 février 1840 à l'Académie et qui servirent à illustrer l'Atlas du cours de microscopie. Pour cela, il fallut mettre au point le microscope en plaçant un écran de verre bleu foncé pour arrêter la chaleur rayonnante, se servir d'une source photoélectrique par incandescence de charbon sous l'influence de courant électrique et adjoindre de multiples appoints avant d'obtenir l'appareil qu'Alfred Donne appela " microscope photoélectrique ".

Alfred Donne fut l'un des premiers médecins chercheurs, un des premiers à appliquer les sciences exactes et les techniques en médecine. Son cours de microscopie (1844) et son Atlas (1845) illustré de daguerréotypes restent parmi les livres princeps. C'est ainsi qu'il étudia le sang et reconnut la véritable nature des globules : globules rouges, globules blancs et globulins (plaquettes). Dans sa polémique avec Raspail qui défendait la thèse de globules albumineux dans le sang, Alfred Donne rétorquait : "les globules du sang ne sont point, comme le dit M. Raspail, un simple précipité d'albumine qui se redissout ou qui se reforme suivant la plus ou moins grande quantité d'eau que $\mathrm{m} / \mathrm{s} n^{\circ} 10$, vol. 11 , ortobre 95 contiennent les vaisseaux. Les globules du sang sont pour moi des petits corps de forme lenticulaire composés d'un tissu, d'un canevas, si je puis dire aussi de fibrine, dans les mailles desquels de l'albumine et de la matière colorante sont déposées". Il décrit encore "les globules lenticulaires devenant sphériques à l'instant même où l'on verse dans le sang une certaine quantité d'eau, en même temps que leur diamètre diminue. Il est probable que dans ce cas le tissu de fibrine absorbe de l'eau qui renfle, et de cette manière il perd sa forme aplatie pour s'arrondir dans tous les sens".

Certains reconnaissaient la valeur du microscope mis au point par Alfred Donne mais doutaient de son utilité, comme De Commercy qui écrivait en 1837 dans son ouvrage Recherches expérimentales sur le sang humain que "des résultats très remarquables ont été obtenus avec le microscope ; mais ces résultats sont plus propres à piquer la curiosité qu'à rendre de grands services à la science ". Alfred Donne, véritable pionnier, appliqua néanmoins ces méthodes à la pathologie. Il décrivit la première observation de leucémie. Le Dr Barth avait examiné une femme de 44 ans admise le 26 juin 1839 à l'Hôtel Dieu dans le service du Pr. Chomel. Elle avait un état de cachexie progressif depuis un an. Une grosse tumeur persistait après l'accouchement d'un enfant mortné dix-sept mois auparavant, reconnue comme étant une splénomégalie. Prise de diarrhée avec des selles sanguinolentes, éprouvant des douleurs abdominales, elle succomba le 14 juillet 1839 et l'autopsie pratiquée 23 heures plus tard reconnut une rate pesant $3 \mathrm{~kg}$ et un foie volumineux. Un échantillon de sang collecté sur le cadavre fut envoyé le jour même à Alfred Donne qui répondit le 17 juillet 1839 : “Le sang que vous $m$ 'avez adressé, mon cher confrère, offre une altération remarquable des plus manifestes, quoique recueilli dans des circonstances défavorables, c'est-à-dire sur un cadavre. Il contient plus de la moitié de globules muqueux. Eh bien, cette espèce de globules blancs ou muqueux domine à un tel point dans le sang que vous m'avez envoyé qu'il y aurait lieu de douter, ne connaissant pas l'observation, si ce sang ne contient pas de pus. Car vous savez que, malheureusement, les globules blancs ne se distinguent pas encore à des caractères certains des globules muqueux. Mais le fait est que les globules muqueux de ce sang, indépendamment de leur nombre, se comportent avec l'ammoniaque comme s'il y avait du pus. "Cette observation intitulée "Leucocytes spléniques diagnostiqués après la mort ; hypertrophie de la rate et du foie ; diarrhée sanguinolente ; autopsie " fut lue à la Société Médicale des Hôpitaux le 28 novembre 1855 alors qu'elle avait été écrite en 1839. Il s'agit probablement de la première observation de leucémie aiguë. Cependant, le fait de n'avoir mentionné cette observation qu'en 1855 et de ne l'avoir publiée qu'en 1856 fit que "les deux médecins français laissèrent échapper une découverte qu'ils tenaient entre leurs mains" (Isambert).

Une autre preuve de la connaissance de cette maladie se trouve dans le cours de microscopie complémentaire aux études médicales publié par Alfred Donne en $1844 \mathrm{chez}$ Baillière. Cette observation est rapportée dans le chapitre "de l'altération des globules blancs". "Il y a donc des cas dans lesquels les globules blancs paraissent en excès dans le sang ; j'ai vérifié ce fait un trop grand nombre de fois, il est trop évident chez certains malades pour que je puisse concevoir le moindre doute à cet égard. En procédant comme je l'ai indiqué à propos des globules blancs, on voit apparaître une si grande quantité de ces globules que les observateurs les moins exercés en sont frappés. Cette circonstance s'est encore récemment offerte à moi chez un malade du service de M. Rayer de l'Hôpital de la Charité. Cet homme dans la force de l'âge était atteint d'une artérite qui affectait spécialement les vaisseaux des membres inférieurs : les deux jambes étaient le siège d'ecchymoses, de phlyctènes gangreneuses, etc. Le sang du malade présentait une telle quantité de 
globules qu'en raison même de la nature de son affection j'ai été porté à croire que le sang était réellement mêlé de pus ; mais, en définitive, il ne me fût pas possible de constater une différence tranchée entre ses globules et les globules blancs. En effet, on ne trouva à l'autopsie aucune trace de pus dans les vaisseaux dans l'intérieur des caillots. En me rappelant que j'avais fréquemment observé un état analogue dans le sang d'individus sans présence de pus, je suis porté à croire que l'excès de globules blancs tient à une sorte d'arrêt dans l'évolution du sang. D'après la théorie que j'ai donnée de l'origine et du mode de transformation des globules sanguins, la surabondance des globules blancs ne serait que le résultat d'un arrêt de développement dans ces particules transitoires. " N'est-ce pas la description de la leucémie avec l'état d'arrêt de maturation des globules blancs?

Pourtant les hématologues actuels (figure 1) rapportent la première description de la leucémie aiguè à deux médecins, l'un anglais Bennett, l'autre allemand Virchow. En effet, le journal Edimbourg Medical and Surgical Journal du $1^{\text {er }}$ octobre 1845 sous le titre "Two cases of disease and enlargement of the spleen in which death takes place from the presence of purulent mater in the blood"publia les observations de deux médecins de Grande-Bretagne, recueillies l'une par Craig en février 1841, l'autre par Bennett en février-mars 1845. Les deux cas sont superposables avec une "sécrétion de lymphe " et "présence de matières purulentes mélangées à du sang faisant succomber le malade". Le médecin écossais concluait en parlant d'une maladie purulente du sang. Quatre semaines après cette publication, Virchow, un auteur allemand, rapportait une observation de "sang blanc " avec un cas similaire ayant une grosse rate. Virchow pensait que la grosse rate était en rapport avec la maladie ainsi que les troubles hémorragiques. Cependant, à l'inverse de Bennett, il ne prit pas le sang blanc pour du pus,

ceux du sang. Il s'agissait pour lui d'une réelle et nouvelle maladie du sang.

Une lutte vive d'antériorité s'entama entre Bennett et Virchow, oubliant totalement Alfred Donne. Le premier appelait cette maladie leucocytaemia (Bennett), l'autre leukoemic ( $V$ irchow). Plus tard Leudet, médecin français, modifia euphoniquement cette dernière dénomination en leucémie. La polémique se poursuivit pour savoir s'il s'agissait de pus ou si la maladie était due à une anomalie des globules. Bennett oublia progressivement dans ses écrits suivants son hypothèse initiale d'une origine purulente de l'hyperleucocytose.

Pour Alfred Donne, tout cela paraissait évident : cet état d'augmentation des globules blancs, entraînant la mort, était phénomène courant ("j’ai vérifié ce fait un trop grand nombre de fois"). Cela ne méritait pas de grande déclaration devant les instances scientifiques. Il l'enseignait dans ses cours et cela était suffisant. La question pour lui aussi était de savoir s'il s'agissait de pus ou de maladic du sang. Il tranche nettement sur cette question en raison de l'absence de source d'infection et de trace de pus dans l'organisme, et parle d'arrêt de développement des globules. Cette idée ne sera pas encore totalement admise à la fin du siècle puisque l'on chercha pendant longtemps les germes responsables et qu'il fallut attendre la thèse de Guillermet (1890), élève de Bard à Lyon, pour parler de cancer propre du sang.

Bien d'autres apports scientifiques et techniques sont dus à Alfred Don-

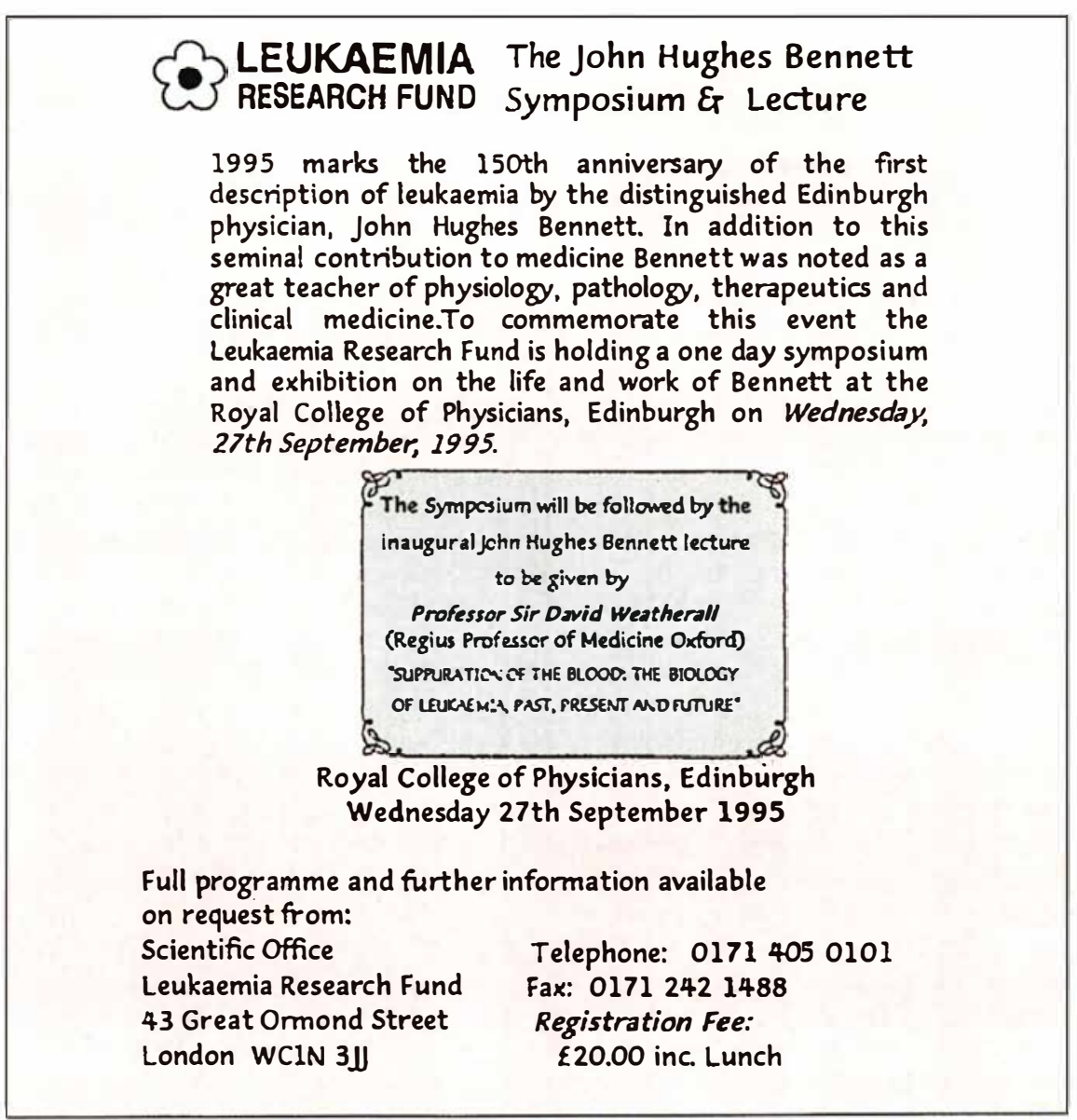

Figure 1. Pour beaucoup de scientifiques, J.H. Bennett reste encore celui qui a découvert la leucémie alors qu'Alfred Donne l'avait déjà décrite. 
ne, comme la première description du Trichomonas vaginalis, la composition microscopique et chimique du lait, la fabrication d'un lactoscope qui mesurait l'opacité du lait et, par conséquent, la quantité d'éléments solides en suspension, soumis à l'Académie le 29 septembre 1843 , ou l'invention d'un appareil pour conserver le lait grâce au refroidissement. Si son engouement à mêler les sciences et techniques à la médecine lui valut une certaine méfiance de la part des médecins, notamment de la faculté de Médecine de Paris, il eut pourtant une carrière tout à fait honorable, souvent soutenu par l'Académie des Sciences. Assurant régulièrement sa chronique dans le Journal des Débats, il lui était reproché de " rehausser les travaux de ses amis ou des amis de ses amis plus fréquemment que ceux des personnes qui n'ont pas l'honneur de vivre dans la sphère où il a le talent de se placer" (Sachalle de la Barre, 1845). Le hasard le mit en contact avec Saint-Marc Girardin, ami de la Famille royale. Le Comte de Paris, âgé de 2 ans, avait une maladie qui avait résisté aux soins de tous les grands médecins de Paris. Le Duc d'Orléans, ayant eu connaissance des travaux d'Alfred Donne et de son esprit scientifique, lui donna toute liberté pour traiter cet enfant abandonné des médecins. Alfred Donne était obsédé par les qualités du lait et l'effet thérapeutique de celui-ci. Après avoir analysé, pesé, " et même dégusté ", le lait de nourrices, il fit son choix et traita ainsi ce jeune enfant. L'apport du lait ressuscita l'enfant. Le Comte de Paris retrouva la santé et Alfred Donne fut nommé Chevalier de la Légion d'Honneur. Il resta le médecin et l'ami intime des membres de la Famille d'Orléans et le ministre de l'Instruction Publique en 1845 créa pour lui les fonctions nouvelles d'Inspecteur général de la Médecine en France. En 1848, le Gouvernement républicain supprima l'Inspection générale. Alfred Donne reprit ses fonctions en 1849 puis fut nommé Recteur provincial à Strasbourg où il resta deux ans et, de là, devint Recteur de Montpellier où il se maintint à son poste pendant dix$\mathrm{m} / \mathrm{s} n^{\circ} 10$, vol. 11, octotre 95 neuf ans, jusqu'à sa retraite. Malgré ses hautes fonctions, il conserva son intérêt pour la recherche médicale et la microscopie. Notamment, il proposa des Prix dont la récompense était un microscope pour encourager cette discipline. Il quitta Montpellier à l'âge de 72 ans en raison d'un nouveau décret qui fixait la retraite des recteurs à 70 ans. De retour à Paris, Alfred Donne tomba rapidement malade et mourut le 9 mars 1878 "à peu près oublié de notre génération médicale et scientifique. Sa carrière, qui s'annonçait brillante, ne répondit pas aux promesses du début. Il ne réussit pas à obtenir à Paris la position qu'il jugeait à la hauteur de son mérite" (Louis Figuier, ancien étudiant du cours de microscopie).

Alfred Donne est un pionnier de la médecine scientifique. L'usage du thermomètre en médecine, le dosage de l'acidité et de l'alcalinité dans les différents liquides, l'étude du sang et du lait au microscope, la microphotographie, la première description de la leucémie, l'identification du Trichomonas vaginalis, différents appareils comme le lactoscope, sont autant de contributions d'Alfred Donne, longtemps oublié. Il n'eut comme oraison après sa mort qu'un cours entrefilet dans le journal Montpellier médical: "M. Donne, avant d'appartenir à l'Université, s'était livré à des travaux de physiologie parmi lesquels il faut citer les recherches devenues classiques sur le lait et avait été l'un des initiateurs des études micrographiques en France. La Faculté de Médecine de Montpellier lui avait dû, à plusieurs reprises, la fondation des Prix consistant en microscopes surtout pour encourager les études parmi les élèves. Homme d'esprit, ne se piquant de rien moins que de rigorisme, aimant le monde, $M$. le Recteur Donne apportait dans les réceptions de l'Académie dont il faisait largement les honneurs, comme dans les réunions de nos associations médicales qu'il acceptait volontiers de présider, un humour de bon aloi et une gaieté toute juvénile qui déridaient les plus moroses et dont le souvenir ne se perdra pas de sitôt dans la Société montpellié-

raine. "Il suffit de relire son traité de microscopie et de voir les premiers daguerréotypes de son Atlas, pour être convaincu que M. Donne était déjà un auteur potentiel de médecine/sciences

\section{Laurent Degos}

Professeur en hématologie clinique, Directeur de l'Unité U. 93 de l'Inserm. Hôpital Saint-Louis, 2, place du Docteur-A.Fournier, 75475 Paris Cedex 10, France.

\section{TIRÉS À PART}

L. Degos.

5. CONGRĖS INTERNATIONAL SUR LES ELLÉMENTS TRACE EN MÉDECINE ET BIOLOGIE

UTILISATIONS THÉRAPEUTIOUES DES OLIGO-ELEMENTS

MÉRIBEL France 4-7 Février 1996

Organisé par La Société Francophone d'Ėtude et de recherche sur les Éléments Trace Essentiels - Sessions : Rôle essentiel et formes thérapeutiques des oligoéléments - La supplémentation en oligoéléments aux différentes périodes de la vie - Oligo-éléments dans les états inflammatoires et infectieux et dans les maladies digestives - Oligo-éléments en endocrinologie - Applications pharmacologiques des oligo-éléments - Oligoéléments, physiologie osseuse et maladies osseuses - Épidémiologie des oligo-éléments et études d'intervention Symposium satellite : désordres génétiques du métabolisme du cuivre.

Information : Arlette Alcaraz, CHRUG Hópital A.-Michallon, Biochimie C, BP 217, 38043 Grenoble Cedex 9, France. Tél: (33) 767654 84. Fax: (33) 76765664. 